\title{
CCD Photometry of RR Lyrae Stars in M3
}

\author{
C. Cacciari ${ }^{1}$, E. Carretta ${ }^{2}$, F. Ferraro ${ }^{1}$, F. Fusi Pecci ${ }^{1}$, G. Tessicini ${ }^{1}$, \\ J.M. Nemec ${ }^{3}$, A.R. Walker ${ }^{4}$ \\ ${ }^{1}$ Bologna Observatory, Italy, ${ }^{2}$ University of Padova, Italy, ${ }^{3}$ Washington State \\ University, Pullman, USA, ${ }^{4}$ University of British Columbia, Victoria, Canada
}

\begin{abstract}
New CCD BVI observations of RR Lyrae variables in M3 are presented. Mean magnitudes and colours are derived, as well as their relations with periods and amplitudes, and comparisons are made with previous data (Sandage 1981, 1990). Preliminary results are presented on the temperature distribution of the variables and the period-shift effect with respect to M15 and M68.
\end{abstract}

\section{Introduction}

The globular cluster M3 has traditionally been assumed as the typical Oosterhoff type-I cluster, and therefore it has been used as the reference cluster for all problems related to globular clusters in general and RR Lyraes in particular, e.g. Oosterhoff dichotomy, period-shift effect, etc.

The data base, however, is still the $\mathrm{B}$ and $\mathrm{V}$ photographic photometry by Roberts and Sandage (1955), Baker and Baker (1956) and Sandage (1959), later re-analysed by Sandage $(1981,1990)$. The need was felt for more recent and accurate observations, in order to verify the assumptions and conclusions derived during the past decade. A project was thus undertaken by Buonanno et al. $(1986,1992)$ with the aim of obtaining photographic and CCD BVI accurate photometry and an independent absolute photometric calibration, as well as infrared JHK CCD photometry, and hence the best possible information on all parts of the CMD.

Different topics (i.e. characteristics and morphologies of the various branches in the CMD) will be treated elsewhere. Here we focus on the RR Lyrae stars only.

\section{The Data Base}

Our data have been collected at two different sites:

LOIANO: $152 \mathrm{~cm}$ telescope $+\mathrm{RCA} 320 \times 512$ pixels, scale $=0.5 \mathrm{arcsec} / \mathrm{px}, \mathrm{FOV}=$ 2'40" $\times 4$ '16", two non-overlapping fields (NW and SE) were observed. 65 frames were taken in each colour (BVI) covering the NW field, and 69 frames in each colour were taken on the SE field. Of these, respectively 44 and 53 have been reduced. A total of 95 variables have been measured, for several of them new or improved periods have been determined. Here we present the preliminary results 


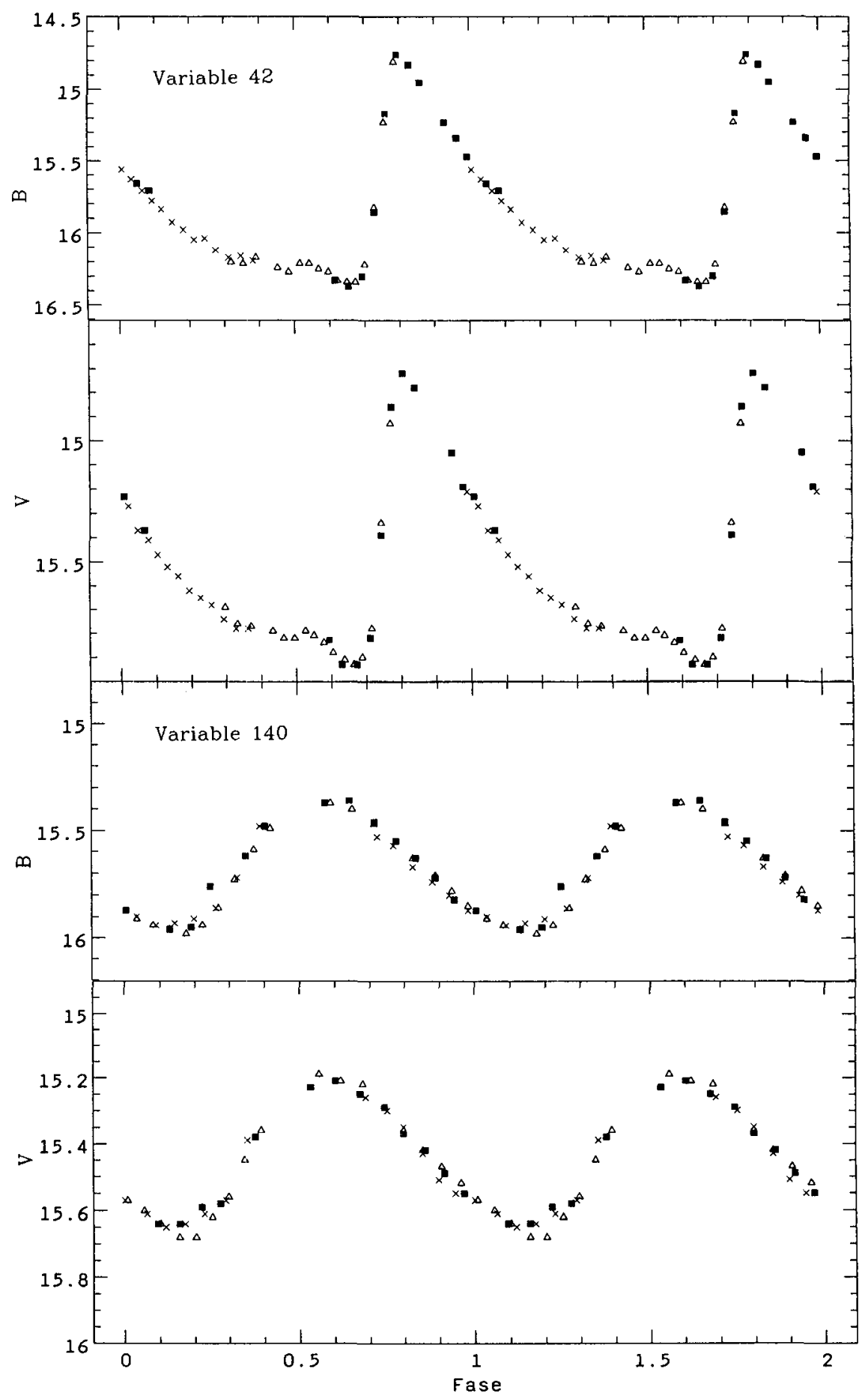

Figure $1 \mathrm{~B}$ and V light curves for the variables V42 and V140 in M3. 
only for the best 38 stars that were measured in the NW field, based on 44 frames (i.e. the data base is still incomplete at the present time). The absolute calibration, based on 15 Landolt standard stars plus 6 stars in M92, has been performed for the NW field only, the calibration work is still in progress for the SE field. The photometric measurements were obtained using the reduction packages ROMAFOT and DAOPHOT, optimized for working in crowded areas, and the mean estimated rms error on the single measure are $\sim 0.01-0.02$ mag.

PALOMAR: $152 \mathrm{~cm}$ telescope $+\mathrm{RCA} 800 \times 800$ pixels (rebinned down to $400 \times 400$ $\mathrm{px}$ ), scale $=0.9 \operatorname{arcsec} / \mathrm{px}, \mathrm{FOV}=66^{\prime} 16^{\prime \prime}$ square, four overlapping fields (NE, NW, $\mathrm{SE}, \mathrm{SW}$ ) were observed. Most frames were aimed at detecting Blue Straggler stars (and actually several variable Blue Stragglers have been found), only about 1520 frames per colour (B and V) per field are suitable for RR Lyrae studies. 137 known or candidate variables have been measured. The calibration of these data is based on Sandage and Katem (1982) photometry, and the data reduction has been performed using the package DoPHOT, which is faster than DAOPHOT but slightly less accurate in crowded areas, the mean estimated rms error on the single measure being $\sim$ 0.05-0.06 mag.

In Figure 1 we show the $B$ and $V$ light curves of the variables 42 and 140 as an example. The preliminary results on our new photometric calibration confirm Sandage (1981, 1990) data within 0.01-0.02 mag: the possibility of a blue shift by $\sim 0.02$ mag in $\mathrm{B}-\mathrm{V}$ cannot be ruled out at present, but needs confirmation from the final calibration work.

A detailed study of the HB morphology will be made when the complete sample of variable and non-variable HB stars will be available. Some information on the period-shift effect, however, can be derived also using the partial sample of stars presented here.

\section{The Colour-Temperature Transformation}

The "Period-Shift Effect" (PSE) means that the period-temperature relations in clusters of different metallicities are shifted as a function of metallicity, in the sense that more metal-poor clusters have tendentially longer periods for the same temperatures. Therefore colours must be transformed to temperatures in order to investigate this issue. However, several problems, both observational and theoretical, affect this transformation:

1. Definition of the "best" mean colour. There are several possibilities: $<\mathrm{B}-$ $\left.\mathrm{V}\rangle,\langle\mathrm{B}\rangle-\langle\mathrm{V}\rangle,(\mathrm{B}-\mathrm{V})_{e q}=2 / 3<\mathrm{B}-\mathrm{V}\right\rangle+1 / 3(\langle\mathrm{~B}\rangle-\langle\mathrm{V}\rangle),(\mathrm{B}-\mathrm{V})_{S}=$ $\langle\mathrm{B}\rangle-\langle\mathrm{V}\rangle+\mathrm{C}\left(\mathrm{A}_{B}\right)$ (Sandage 1990). These same types of indices can be defined for V-I, or for any other colour. They are all affected by some degree of inaccuracy, the effect being strongest on bluer colours, since they average LTE with non-LTE (shock waves at maximum light) values. The colours $<$ V$\mathrm{K}\rangle$ or $\langle\mathrm{V}\rangle-\langle\mathrm{K}\rangle$ are in principle better temperature indicators since they 
have a longer baseline, are less affected by non-LTE problems and are less sensitive to metallicity.

Finally, another question which affects the definition of the "best" mean colour is: intensity or magnitude means?

2. From the observational point of view, the reddening represents a serious problem: as we shall see later, even a difference of $0.02-0.03 \mathrm{mag}$, which is of the same order as the accuracy of most reddening determinations, may make a significant difference in the analysis and interpretation of the PSE (Caputo 1987).

3. The temperature scale.

a) By definition, the equilibrium temperature of a star is given by the StefanBoltzmann law, but radius and luminosity must be known. For RR Lyrae stars this is presently possible only for the $\sim 25$ stars analysed with the Baade-Wesselink method (Jones et al. 1992; Cacciari, Clementini and Fernley 1992), but there may be some problem with the zero-point of the B-W results, which appears to be $\sim 0.2 \mathrm{mag}$ too faint, as suggested by several authors also in this conference (e.g. Fernley, Saha, Cacciari, Dorman, Freedman, Longmore, Walker).

b) A relation was found between equilibrium temperature, pulsation period, metallicity and amplitude of the $B$ light curve (Carney, Storm and Jones 1992), which however does not appear to be very accurate.

c) A different set of problems is related to the use of purely theoretical models (Kurucz, Bell-Gustafsson, VandenBerg-Bell, etc.). Kurucz models, for example, have been found to be somewhat incorrect, especially in the bluest part of the spectrum, and possibly in correlation with metallicity.

d) Empirical scales can be used as an alternative: however, they are derived only for Population I main sequence stars where there is a reasonable number of stars with measured angular diameters. For more evolved or more metal-poor stars the transformations make use again of model atmospheres differentially.

The problem of a correct temperature determination is a very important one, but will not be addressed here in any further detail. For the sake of comparison with Sandage's previous work on M3 and with other clusters, we shall use consistently the colours $\langle\mathrm{B}\rangle-\langle\mathrm{V}\rangle+\mathrm{C}\left(\mathrm{A}_{B}\right)$ and Kurucz models, and $\langle\mathrm{V}\rangle$ $-<\mathrm{K}>$ and Fernley (1989) calibration when infrared colours are available.

\section{The Period-Shift Effect}

First we compare the present data (using only the 38 variables we have accurately measured in the NW field of M3) with the previous data obtained 
by Sandage $(1981,1990)$ in the $\log P-\log T_{e}$ plane. The B-V colours are actually $\langle\mathrm{B}\rangle-\langle\mathrm{V}\rangle+\mathrm{C}\left(\mathrm{A}_{B}\right)$, where $\mathrm{C}\left(\mathrm{A}_{B}\right)$ is determined according to Sandage (1990), and the Kurucz models have been interpolated for the cluster metallicity and $\log g=2.75$. The reddening for M3 has been assumed to be zero. Figure 2 shows our data and, superimposed, the solid line representing the best fit to Sandage (1990) data. The two distributions are consistent, and would coincide if our data were red-shifted by about $0.02 \mathrm{mag}$. The same indication comes from the comparison with the M5 data using the mean V magnitudes by Storm et al. (1991) and Goranskj (1982), assuming $E(B-V)=$ 0.03 for M5. Since our calibration is still in progress, we provisionally apply a red-shift of 0.02 mag to our colours.

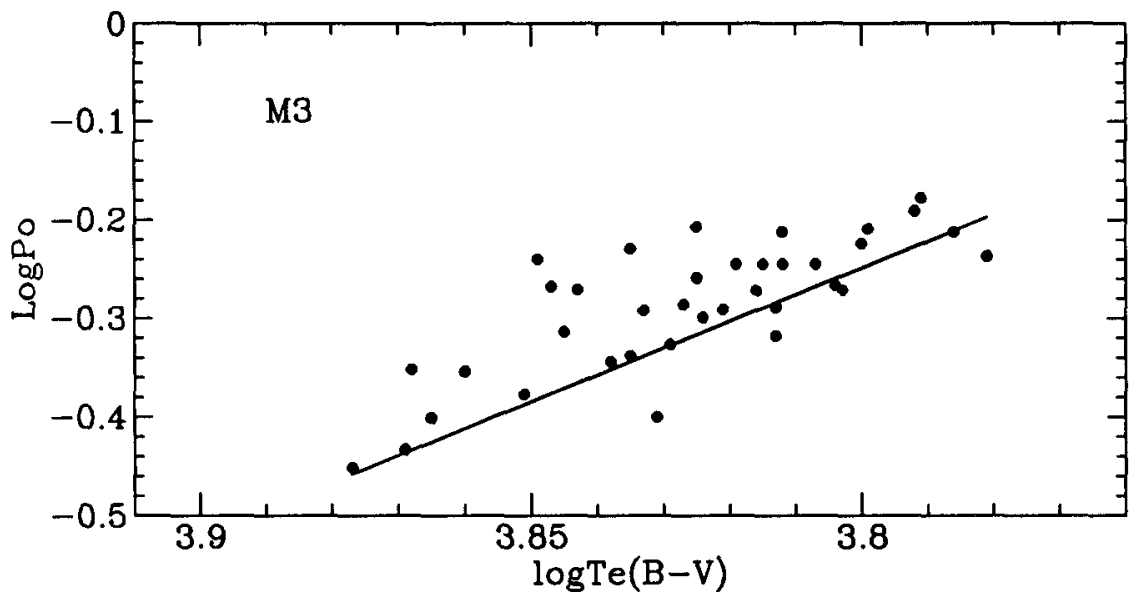

Figure 2 Data points from our new photometry. The $\log \mathrm{P}_{0}$ represents the fundamental period ( 0.125 was added to the c-type variables). The solid line is the best fit to Sandage (1990) data.

If we now compare the M3 data with the data obtained for M15 using Bingham et al. (1984) photometry and $E(B-V)=0.10$, and with M68 using unpublished photometry by Andrews and $E(B-V)=0.03$, we find a period shift $\Delta \log \mathrm{P} / \Delta[\mathrm{Fe} / \mathrm{H}] \sim-0.12$ and -0.07 respectively. These values would coincide (i.e. -0.07) if the reddening for M15 were 0.07 , as suggested by a number of independent studies, especially on UV data.

Since for all these clusters, except M68, K photometry is available (Longmore et al. 1990), it is worth repeating this comparison using $V-K$ colours and Fernley's (1989) semi-empirical calibration:

$$
\log T_{e}=-0.125(V-K)-0.003[F e / H]+3.934
$$


which is valid for $R R$ Lyraes at temperatures around $6500 \mathrm{~K}$ and all metallicities. The smaller slope of the colour-temperature relation decreases the effect of possible errors in the colours and improves the accuracy of the comparison: again the period-shift between $\mathrm{M} 3$ and $\mathrm{M} 15$ leads to $\Delta \log \mathrm{P} / \Delta[\mathrm{Fe} / \mathrm{H}] \sim-0.07$ or -0.12 if a reddening of 0.07 or 0.10 respectively are assumed for M15.

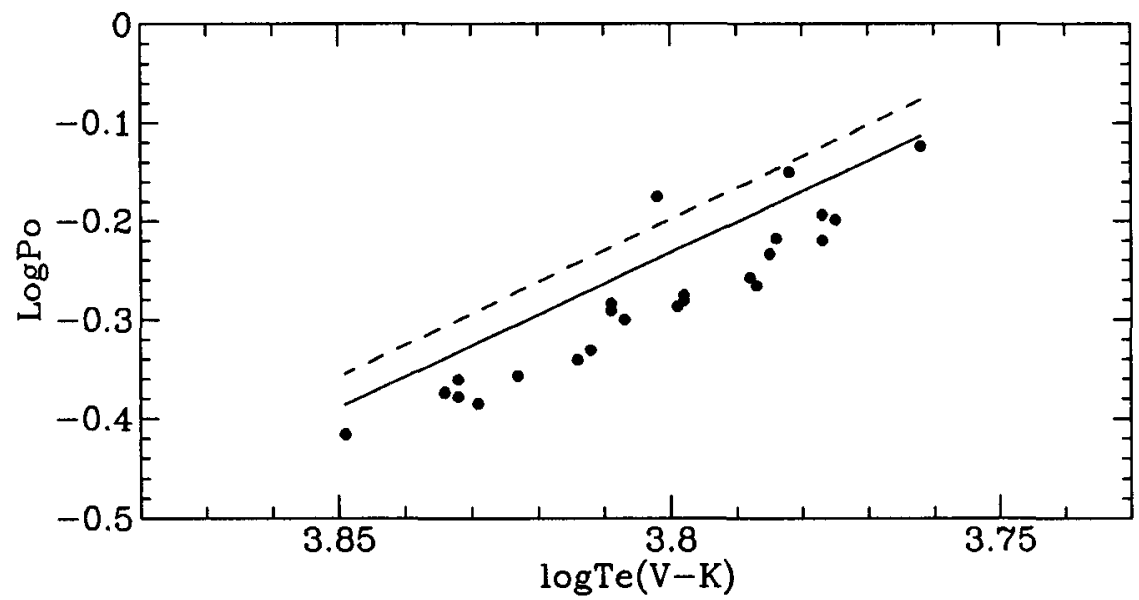

Figure 3 Our data for the variables in M3. The lines represent the best fit to the M15 variables using $E(B-V)=0.10$ (dashed line) and 0.07 (solid line).

\section{Summary and Conclusions}

(a) For the "standard" Oosterhoff I cluster M3 a new photometric data base (CCD BVI) has been obtained. A preliminary independent calibration confirms Sandage $(1981,1990)$ results within about 0.02 mag. Work is still in progress for a final accurate calibration.

(b) Referring to RR Lyraes only: the problem of the PSE depends mainly on: a) the colour - temperature transformation; b) Off-ZAHB evolution; c) the reddening.

(c) The reddening plays a key role: if $\mathrm{E}(\mathrm{B}-\mathrm{V})=0.07$ for $\mathrm{M} 15$, which gives consistent results with other clusters and is suggested by UV studies, then the period-shift between M3 \& M5 and M15 \& M68 is about 0.030.04 . Therefore the period-shift dependence on metallicity is about 0.06 0.07 , as suggested independently by other studies (e.g. Fernley elsewhere in this conference).

If, on the contrary, the reddening of M15 is 0.10 , as suggested by optical studies, then evolution off the ZAHB for most of the RR Lyraes in M15 
could explain the high slope of the period shift-metallicity relation (and hence of the luminosity-metallicity relation), as already suggested by Lee, Demarque and Zinn (1990).

\section{References:}

Baker, R.H., and Baker, H.V., 1956, Astron. J., 61, 283.

Bingham, E.A., Cacciari, C., Dickens, R.J., and Fusi Pecci, F., 1984, M.N.R.A.S. $209,765$.

Buonanno, R., Buzzoni, A., Corsi, C.E., Fusi Pecci, F. and Sandage A., 1986, Mem. Soc. Astr. It. 57, 535.

Buonanno, R., et al., 1992, in preparation

Cacciari, C., Clementini, G., and Fernley, J.A., 1992, Astrophys. J., Sept. 1 issue, in press.

Caputo, F., 1987, in Stellar Evolution and Dynamics in the Outer Halo of the Galaxy, Ed.s M.Azzopardi and F.Matteucci, ESO Conf. Proc. No.27, p.321.

Carney, B.W., Storm, J., and Jones, R.V., 1992, Astrophys. J., 386, 663.

Fernley, J.A., 1989 , M.N.R.A.S., 239, 905.

Goranskij, V.P., 1982, Astr. Tsirk. 1207, 4.

Jones, R.V., Carney, B.W., Storm, J., and Latham, D.W., 1992, Astrophys. J., 386, 646.

Lee, Y.W., Demarque, P. and Zinn, R., 1990, Astrophys. J., 350, 155.

Longmore, A.J., Dixon, R., Skillen, I., Jameson, R.F., and Fernley, J.A., 1990, M.N.R.A.S., 247, 684.

Roberts, M.S., and Sandage, A., 1955, Astron. J., 60, 185.

Sandage, A., 1959, Astrophys. J., 129, 596.

Sandage, A., 1981, Astrophys. J., 248, 161.

Sandage, A., 1990 Astrophys. J., 350, 603.

Sandage, A., and Katem, B., 1982, Astron. J., 87, 537.

Storm, J., Carney, B.W., and Beck, J.A., 1991, P.A.S.P. 103, 1264.

\section{Discussion}

COX: Could the -0.07 coefficient of the period shift - metallicity relation be within the errors of the Sandage value near -0.1 ?

CACCIARI: As I said before, the effect of reddening is quite important on this issue, and reddening is not determined with a precision better than $0.02-$ $0.03 \mathrm{mag}$, especially when it's large. This error is sufficient to change the coefficient from -0.12 to -0.07 even in the case of M15, whose reddening is not very high.

FERNLEY: I have a comment: just to add another piece to the issue I find a slope $d \log \mathrm{P} / \mathrm{d}[\mathrm{Fe} / \mathrm{H}]=-0.07$ from field $\mathrm{RR}$ Lyrae stars (see talk in these proceedings). 